\title{
Study of the response of the penile corporal tissue and cavernosus muscles to micturition
}

\author{
Ahmed Shafik*1, Ismail A Shafik ${ }^{1}$, Olfat El Sibai ${ }^{2}$ and Ali A Shafik ${ }^{1}$
}

Address: ${ }^{1}$ Department of Surgery and Experimental Research, Faculty of Medicine, Cairo University, Cairo, Egypt and ${ }^{2}$ Department of Surgery, Faculty of Medicine, Menoufia University, Shebin El-Kom, Egypt

Email: Ahmed Shafik* - shafik@ahmedshafik.com; Ismail A Shafik - shafik@ahmedshafik.com; Olfat El Sibai - shafik@ahmedshafik.com; Ali A Shafik - shafik@ahmedshafik.com

* Corresponding author

Published: 2 March 2008

BMC Urology 2008, 8:4 doi:10.1 186/147|-2490-8-4

This article is available from: http://www.biomedcentral.com/I47I-2490/8/4

(C) 2008 Shafik et al; licensee BioMed Central Ltd.

This is an Open Access article distributed under the terms of the Creative Commons Attribution License (http://creativecommons.org/licenses/by/2.0), which permits unrestricted use, distribution, and reproduction in any medium, provided the original work is properly cited.
Received: 15 November 2007

Accepted: 2 March 2008

\begin{abstract}
Background: The reaction of the corpora cavernosa (CC), the corpus spongiosum (CS), the bulbocavernosus (BCM) and ischiocavernosus (ICM) muscles to passage of urine through the urethra during micturition is not known. We investigated the hypothesis that the passage of urine through the urethra stimulates the corporal tissue and cavernosus muscles.

Methods: In 30 healthy men (mean age $42.8 \pm$ I I.7 years), the electromyographic activity (EMG) of the CC, CS, BCM, and ICM were recorded before and during micturition, and on interruption of and straining during micturition. These tests were repeated after individual anesthetization of urethra, corporal tissue, and cavernosus muscles.

Results: During micturition, the slow wave variables (frequency, amplitude, conduction velocity) of the CC and CS decreased while the motor unit action potentials of the BCM and ICM increased; these EMG changes were mild and returned to the basal values on interruption or termination of micturition. Micturition after individual anesthetization of urethra, corporal tissue and cavernosal muscles did not effect significant EMG changes in these structures, while saline administration produced changes similar to those occurring before saline administration.

Conclusion: The decrease of sinusoidal and increase of cavernosus muscles' EMG activity during micturition apparently denotes sinusoidal relaxation and cavernosus muscles contraction. Sinusoidal muscle relaxation and cavernosus muscles contraction upon micturition are suggested to be mediated through a 'urethro-corporocavernosal reflex'. These sinusoidal and cavernosus muscle changes appear to produce a mild degree of penile tumescence and stretch which might assist in urinary flow during micturition.
\end{abstract}

\section{Background}

The mechanism of micturition is intricate and under the control of reflex and voluntary actions [1-7]. Various reflexes are involved in facilitating or inhibiting this mechanism [8-11]. The micturition reflex is apparently the main reflex. The urinary bladder, bladder neck, ure- thra, and external urethral sphincter (EUS) exhibit a wellcoordinated behaviour during continuous filling of the urinary bladder [12]. During voluntary voiding, the intravesical pressure increases while the intraurethral pressure decreases and the EUS relaxes. 
The penile urethra passes through the corpus spongiosum (CS) which consists of sinusoids. The sinusoids of the corpora cavernosa (CC) and corpus spongiosum (CS) of the penis are contracted and contain a minimal amount of blood in the flaccid phase, while they relax and are full of blood during erection. The bulbo- and ischio-cavernosus muscles (BCM, ICM) contract in the rigid erectile phase $[13,14]$. The recording of the electric activity of the corporal tissue (CC, CS) was introduced by Wagner and Gerstenberg in 1989 [15] and later studied by other investigators [16-20].

The reaction of the CC, CS or the cavernosus muscles to the passage of urine in the urethra during micturition is not known. We hypothesized that passage of urine in the urethra during micturition stimulates the corporal tissue and cavernosus muscles. This hypothesis was investigated in the current study.

\section{Methods}

Subjects

Thirty healthy men (mean age $42.8 \pm 11.7$ SD years, range 29-52) volunteered for the study. They were recruited from our University Hospital workers who were paid. They had no genitourinary complaint in the past or at the time of enrollment. They signed an informed consent after they had been notified about the protocol of the study.

Laboratory work, including urinalysis, blood cell count, liver and kidney function tests as well as electrocardiography showed normal findings. Also, the sonogram of the urinary tracts were normal. The Cairo University Faculty of Medicine Review Board and Ethics Committee approved the study.

\section{Methods}

The subjects were asked to empty their bladders prior to performing the tests. Electromyographic (EMG) electrodes were applied to the penile cavernosus tissue and to the BCM and ICM, and their basal EMG activity was recorded. Full bladder was achieved by asking the subject to continuously drink water until he felt the desire to micturate. The subject was then allowed to micturate. During micturition he was asked to strain, to interrupt micturition and then to evacuate the bladder, while the EMG response of the cavernosus tissue, BCM and ICM was being registered.

The EMG activity was recorded by means of a concentric EMG needle electrode (Type 13 L 49, DISA, Copenhagen, Denmark) measuring $40 \mathrm{~mm}$ in length and $0.65 \mathrm{~mm}$ in diameter. Two needle electrodes were introduced into each of the corpus cavernosus (CC) and corpus spongiosum (CS): one in the upper and one in the lower third. A ground electrode was applied to the thigh and a straingauge respiratory transducer to the thoracic wall. After recording the electric activity, the upper electrode was transferred to the mid third of the CC and the CS, respectively, and the recordings were repeated. The needle electrodes were then transferred to the contralateral CC and the electric activity was recorded.

The EMG activity of the ICM and BCM was recorded by means of a concentric EMG needle electrode (type 13L49, DISA, Copenhagen) measuring $40 \mathrm{~mm}$ in length and 0.65 $\mathrm{mm}$ in diameter. The ischiopublc ramus of the ICM with the overlying crus penis was palpated and the needle inserted into the ICM lying on its medial aspect. A second identical needle was placed in the BCM; the penile bulb was palpated and the needle electrode introduced into the muscle overlying it.

A standard EMG apparatus (Type MES, Medelec, Woking, UK) was used to amplify and display the recorded potentials. Films of these potentials were taken on a light-sensitive paper (Linagraph type 1895, Kodak, London, UK) from which measurements of the duration of the motor unit action potentials (MUAPs) were obtained. The EMG signals were in addition stored on an FM tape recorder (type 7758A, Hewlett-Packard, Waltham, MA) for further analysis as required. All filtered signals were collected and recorded using an online computer with data acquisition and analysis software (Chart V 4.2, AD instruments, Castle Hill, Sydney, Australia). The acquisition rate was $10 \mathrm{~Hz}$, and the EMG normal band width was 0.1 to $5.0 \mathrm{~Hz}$.

After micturition, erection was induced by electrovibration [21], and the EMG activity of the CC, CS, BCM, and ICM during erection was registered.

\section{Urethral, CC, CS, BCM, and ICM anesthetization}

To examine whether the effect of urethral stimulation, which is produced by the urine passage through the urethra, on the CC, CS, BCM, and the ICM was a direct or reflex effect, the urethra was anesthetized by administration of 5\% xylocaine gel (Astra, Södertälje, Sweden). The gel was introduced into the urethra through the gel container nozzle after the urethral orifice had been sterilized by alcohol. Twenty minutes after urethral anesthetization, the EMG activity of the $\mathrm{CC}, \mathrm{CS}, \mathrm{BCM}$, and ICM was recorded during micturition, on straining during micturition and on micturition interruption. The readings were also taken three hours later when the anesthetic effect had waned. The test was repeated using bland gel instead of xylocaine gel.

On sepate days, each of the BCM, ICM, CC, CP was individually anesthetized and the effect of urine flow through the urethra on these structures was recorded. Two $\mathrm{ml}$ of $2 \%$ xylocaine were injected into each of the CC, CS, BCM, ICM around the inserted needle electrode; the response of these 
structures to urine flow through the urethra was then recorded 20 minutes from injection and again 3 hours later. The test was repeated using normal saline instead of xylocaine.

To ensure reproducibility of the results, the recordings were repeated at least twice in the individual subject and the mean value was calculated. The results were analysed statistically using the paired Student's t test, and values were given as the mean \pm SD. Significance was ascribed to $\mathrm{p}<0.05$.

\section{Results}

No adverse side effects were encountered during or after performing the tests and all the subjects were evaluated and the tests completed.

\section{Electrocavernosogram of the CC and CS}

Basal slow waves (SWs) were recorded from the electrodes applied to the CC and CS (figs. 1, 2). The waves were positive deflected (figs. 1, 2) and had an invariable shape in all

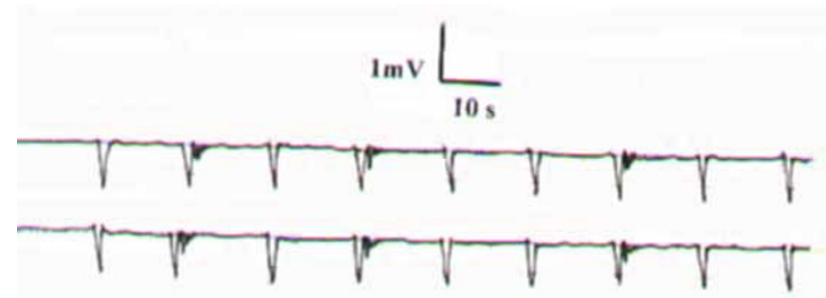

Figure I

The basal slow waves and action potentials recorded from the corpus cavernosum. They have the same frequency, amplitude, and conduction velocity from the two electrodes. the recordings from the same site. In each individual, they exhibited the same frequency, amplitude, and conduction velocity from the 2 electrodes and were constant. The SW variables for the CC and CS are shown in table 1. It is to be observed that the SW variables of the CS exhibited significantly lower values than those of the CC. (figs. 1, 2, table 1). Bursts of fast activity spikes or action potentials (APs) were superimposed on or followed the SWs. They presented as negative deflections and their frequency was inconsistent in each subject (figs. 1,2).

The BCM and ICM displayed no basal electric activity (fig. 3); no MUAPs were recorded.

During bladder filling, the EMG activity levels of the CC, CS, BCM, and ICM did not differ from those of the basal ones. However, with the start of micturition, the SW variables of CC and CS decreased, while the MUAPs of BCM and ICM increased ( $\mathrm{p}<0.05$, figs. 3, 4, tables 1, 2). These changes of the wave variables and MUAPs remained constant during the period of micturition. When the urine flow was interrupted, the EMG of the CC, CS, BCM, and

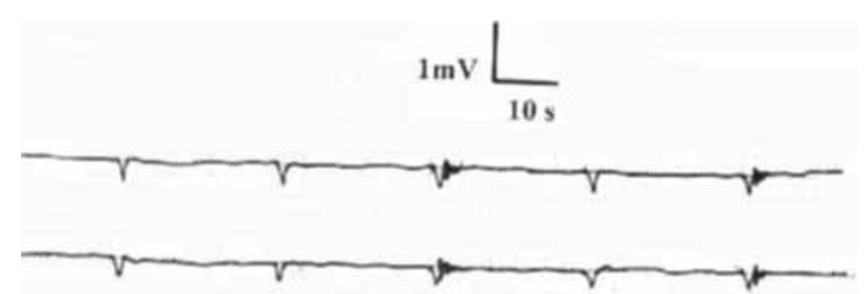

Figure 2

The basal slow waves and action potentials recorded from the corpus spongiosum of the same subject of figure $I$.

Table I: The frequency, amplitude, and conduction velocity of the slow waves recorded from the corpora cavernosa (CC) and corpus spongiosum (CS) at rest (basal) and during micturition ${ }^{+}$

\begin{tabular}{|c|c|c|c|c|c|c|c|c|}
\hline \multirow[t]{3}{*}{ Slow Waves } & \multicolumn{4}{|c|}{ Basal values } & \multicolumn{4}{|c|}{ During micturition } \\
\hline & \multicolumn{2}{|c|}{ CC } & \multicolumn{2}{|c|}{ CS } & \multicolumn{2}{|c|}{ CC } & \multicolumn{2}{|c|}{ CS } \\
\hline & Mean & Range & Mean & Range & Mean & Range & Mean & Range \\
\hline $\begin{array}{l}\text { - Frequency } \\
\text { (cycle/min) }\end{array}$ & $4.8 \pm 1.3$ & $3.9-6.2$ & $3.6 \pm 1.1$ & $2.7-4.8$ & $2.6 \pm 0.9 *$ & $1.7-3.4$ & $1.2 \pm 0.6 * *$ & $0.9-1.9$ \\
\hline $\begin{array}{l}\text { - Amplitude } \\
\text { (mV) }\end{array}$ & $0.58 \pm 0.06$ & $0.47-0.77$ & $0.42 \pm 0.04$ & $0.31-0.63$ & $0.21 \pm 0.03 *$ & $0.16-0.34$ & $0.19 \pm 0.01 * *$ & $0.12-0.24$ \\
\hline $\begin{array}{l}\text { Conduction } \\
\text { velocity } \\
(\mathrm{cm} / \mathrm{s})\end{array}$ & $5.3 \pm 0.9$ & $4.3-6.2$ & $3.6 \pm 0.6$ & $3.1-4.4$ & $2.6 \pm 0.4^{*}$ & $1.2-3.2$ & $1.4 \pm 0.2^{* *}$ & $0.9-2.2$ \\
\hline
\end{tabular}

+ values were given as the mean \pm SD.

$* \mathrm{P}<0.05$

$* * \mathrm{P}<0.01$ 


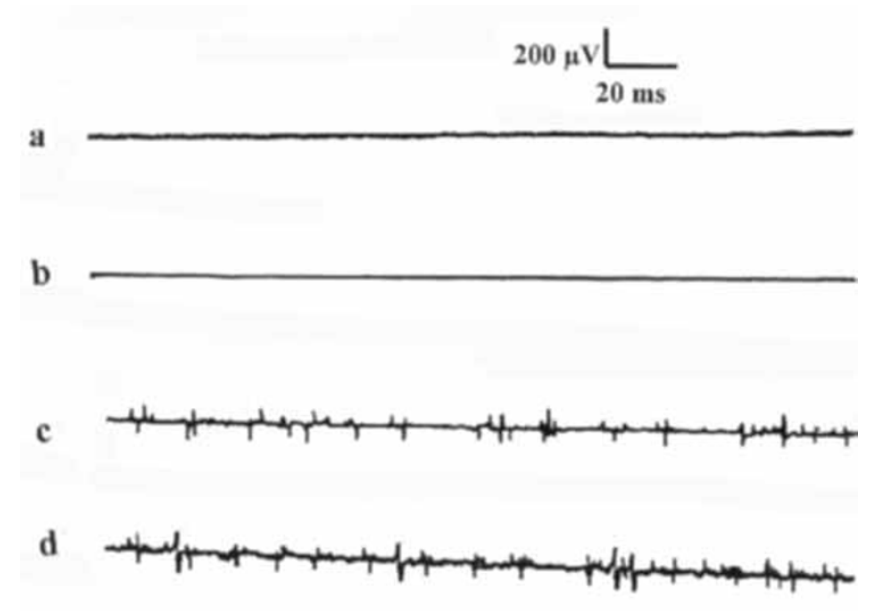

Figure 3

The EMG activity of (a) bulbocavernosus muscle at rest, (b) ischiocavernosus muscle at rest, (c) bulbocavernosus muscle during micturition, and (d) ischiocavernosus muscle during micturition.

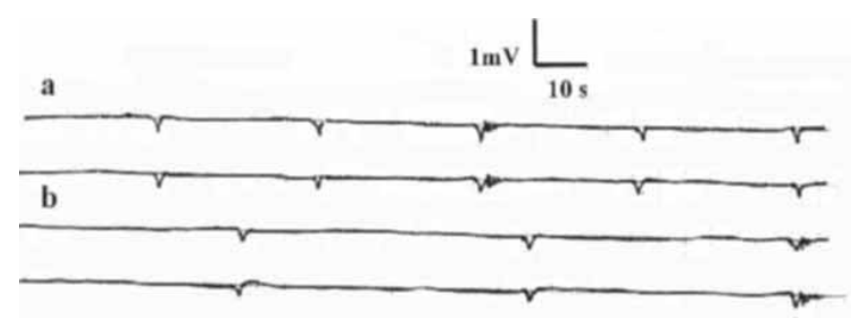

Figure 4

EMG of (A) corpus cavernosum and (B) spongiosum during micturition.

ICM returned to the basal values with no significant difference ( $p>0.05)$. When during micturition the subject was asked to strain in an attempt to increase the urine flow, the EMG activity of the CC, CS, BCM, and ICM did not significantly change from the readings without straining ( $\mathrm{p}>$ 0.05). Upon termination of micturition, the EMG activity of the CC, CS, BCM, and ICM recorded the basal activity $(\mathrm{p}>0.05)$.
When rigid penile erection was induced, the EMG of the CC and CS showed a significant reduction $(\mathrm{p}<0.01)$ to a level lower than the one recorded during micturition. Meanwhile, the cavernosal muscles exhibited a significant increase.

\section{Effect of anesthetization of urethra, corporal tissue and cavernosus muscles}

Micturition 20 minutes after anesthetization of the urethra did not produce the significant changes $(\mathrm{p}>0.05)$ in the EMG of the CC, CS, BCM, and ICM which occurred on micturition as already mentioned. Repetition of the test 3 hours after anesthetization, when the anesthetic effect had waned, produced an EMG response similar to that before anesthetization ( $p>0.05$ ). Micturition 20 minutes after individual anesthetization of the CC, CS, BCM, and ICM did not effect a significant EMG response in these structures. When the anesthetic effect had waned 3 hours from anesthetization, the EMG response of the CC, CS, BCM, and ICM was similar to that before anesthetization. When we repeated the test after administration of bland gel into the urethra or after injection of saline into the $\mathrm{CC}, \mathrm{CS}$, $\mathrm{BCM}$, and the ICM instead of xylocaine, the results were similar to those before gel or saline administration.

The aforementioned results were reproducible with no significant difference when the tests were repeated in the same subject.

\section{Discussion}

In the flaccid phase of erection, the sinusoids contain only a small amount of blood and the smooth muscle fibers are in a state of contraction. In the tumescent stage, the smooth muscle fibers relax and the sinusoids dilate and fill with blood. The smooth muscles surrounding the corporal sinusoids showed in the flaccid phase, a high basal EMG activity indicating that they were in a contracted state [16-20]. Meanwhile, the absence of EMG activity of the cavernosus muscles at rest point to full relaxation of the muscles.

The current study has demonstrated that, during micturition, the urinary flow through the urethra was associated with diminished EMG activity of the smooth muscle fibers of the corporal sinusoids indicating their relaxation. The reduction in the corporal EMG activity was signifi-

Table 2: The motor unit action potentials of the bulbocavernosus (BCM) and ischiocavernosus (ICM) muscles before (basal) and during micturition $^{+}$

\begin{tabular}{cccccc}
\hline & $\operatorname{BCM}(\mu \mathbf{V})$ & & ICM $(\mu \mathbf{V})$ & Mean & Range \\
\hline $\begin{array}{c}\text { Mean } \\
\text { Micturition }\end{array}$ & 0 & Range & 0 & 0 & 0 \\
\hline
\end{tabular}

+ values were given as the mean $\pm \mathrm{SD}$ 
cantly lower than that induced at full erection. Thus, it seems that the passage of urine through the urethra was associated with a small degree of tumescence induced by slight degree of sinusoidal muscles' relaxation. Tumescence disappeared on interruption or termination of micturition as evidenced by return of the EMG activity of the CC and CS to their basal values. The MUAPs of the cavernosus muscles recorded during micturition apparently denote that the muscles are in a state of contractile activity; the recorded EMG activity levels were low compared to those recorded during rigid erection.

The questions that need to be discussed are: what is the effect of the micturition-produced a) mild sinusoidal muscle relaxation and b) mild increase of cavernosus muscles' EMG activity? It appears that the slight sinusoidal relaxation with a resulting mild sinusoidal filling with blood leads to mild penile congestion and stretch. It is known that the penile cavernosus tissue in the flaccid stage is contracted and that the sinusoidal blood volume is scanty. On mild sinusoidal filling with blood during micturition, the penis seems to acquire a degree of tumescence with a resulting mild penile stretch. This penile congestion with stretch seems to effect a degree of penile urethral stretch which might assist in easing the flow of urine through the urethra.

Meanwhile, we do not know the exact role of the mild increase of the EMG activity of the cavernosus muscles during micturition. Does this increase assists in stretching straight the penis by augmenting its blood through tightening the constricting band of the cavernosus muscles situated across the corporal tissue.

Another discussion arising around the process of micturition is whether the response of the CC, CS, BCM, and ICM to urine passing through the urethra is representing a direct or a reflex action. It appeared from our study that the passage of urine through the urethra seems to stimulate the urethral mechanoreceptors which are presumably responsible for the eventually induced effect on the corporal tissue and the cavernosus muscles. This effect is very likely induced through a reflex and not a direct action: the mechanoreceptors seem to transmit the urethral stimuli through the afferent urethral nerves to the spinal cord and then through the efferent nerves to the corporal tissue and cavernosus muscles. The mechanism of action of this assumed reflex needs to be discussed.

\section{The urethro-corporocavernosal reflex}

The EMG response of the corporal tissue and cavernosus muscles to urine traveling through the urethra postulates a reflex relationship between the 2 actions. The constancy of this relationship is indicated by being reproducible. Meanwhile, its reflex nature is evidenced by the absence of the response of the corporal tissue and cavernosus muscles to passage of urine through the urethra upon individual anesthetization of the assumed 2 arms of the reflex arc: the penile urethra as one arm and the corporal tissue/cavernosus muscles as the other arm. We call this reflex relationship the 'urethro-corporocavernosal reflex' (UCCR). It seems necessary to denote that lidocaine does not block the muscle' motor activity but rather the sensory fibers $(\mathrm{C}$ and $A \alpha$-fibers) which are responsible for pain and reflex activity $[22,23]$.

It appears that during micturition the mild sinusoid relaxation and the accompanying mild cavernosus muscles contraction lead to slight penile tumescence and straightening which presumably assist in the passage of the urine through the urethra.

\section{Conclusion}

The decrease of sinusoidal and the increase of cavernosus muscles' EMG activity during micturition apparently denotes sinusoidal relaxation and cavernosus muscles contraction. Sinusoidal muscle relaxation and cavernosus muscles' contraction upon micturition are suggested to be mediated through a 'urethro-corporocavernosal reflex'. These sinusoidal and cavernosal muscles' changes seem to effect a mild degree of penile tumescence and stretch which could assist in urinary flow during micturition. Further studies are warranted to investigate a possible diagnostic significance of this reflex in neurogenic disorders of the urethra or micturition disturbances.

\section{Abbreviations}

corpora cavernosa $=(\mathrm{CC})$, corpus spongiosum $=(\mathrm{CS})$, bulbocavernosus $=(\mathrm{BCM})$ ischiocavernosus $=(\mathrm{ICM})$, electromyographic $=($ EMG $)$, external urethral sphincter $=$ (EUS) slow waves $=($ SWs $)$, action potentials $=($ APs $)$

\section{Competing interests}

The author(s) declare that they have no competing interests.

\section{Authors' contributions}

AS carried out the study design, data collection, statistical analysis, data interpretation and preparation of manuscript. IA participated in data collection and analysis and literature search. OE participated in data collection, statistical analysis and literature search. AAS participated in data collection and preparation of the manuscript. All authors read and approved the final manuscript.

\section{Acknowledgements}

Margot Yehia assisted in preparing the manuscript.

\section{References}

I. Denny-Brown D, Robertson EG: The physiology of micturition. Brain 1936, 56:149-184. 
2. Maggi CA, Meli A: The role of neuropeptides in the regulation of the micturition reflex. J Auton Pharmacol 1986, 6(2):133-137.

3. Mallory B, Steers WD, de Groat WC: Electrophysiological study of micturition reflex in the rat. Am J Physiol 1989, 257(2):410-4I7.

4. Janig W, Koltzenburg M: On the function of spinal primary afferents supplying colon and urinary bladder. J Auton Nerv Syst 1990, 30:89-96.

5. Theobald RJ: Purinergic and cholinergic components of bladder contractility and flow. Life Sci 1995, 56:445-454.

6. Park JM, Bloom DA, McGuire Ej: The guarding reflex revisited. Brit J Urol 1997, 80:940-943.

7. Shafik A: A study of the continence mechanism of the external urethral sphincter with identification of the voluntary urinary inhibition reflex. J Urol 1999, I62:|967-197|.

8. Morrison JFB, Spillane K: Neuropharmacological studies on descending inhibitory control over micturition reflex. J Auton Nerv Syst (Suppl) 1986:393-396.

9. Griffiths D, Holstege G, Dalm E, de Wall H: Control and coordination of bladder and urethral function in the brainstem of the cat. Neurourol Urodynam 1990, 9:63-67.

10. Fedirchuk B, Downie J, Shefchyk SJ: Reduction of perineal evoked excitatory postsynaptic potentials in cat lumbar and sacra motoneurons during micturition. J Neurosci 1994, 14:153-159.

II. Blok BFM, Holstege G: Direct projections from the periaqueductal gray to the pontine micturition center (M-Region). An anterograde and retrograde tracing study in the cat. Neurosci Lett 1994, 166:93-96.

12. Klevmark B: Motility of the urinary bladder in cats during filling at physiological rates I. Intravesical pressure patterns studied by a new method of cystometry. Acta Physiol Scand 1974, 90:565-577.

13. Giuliano F, Rampin O: Central neural regulation of penile erection. Neurosci Biobehav Rev 2000, 24:56I-569.

14. Blewstein CB, Arezzo JC, Eckholdt H, Melman A: The neuropathy of erectile dysfunction. Int J Impot Res 2002, 14:433-439.

15. Wagner G, Gerstenberg T, Levin RJ: Electrical activity of corpus cavernosum during flaccidity and erection of the human penis: a new diagnostic method? J Urol 1989, 142:723-725.

16. Stief CG, Djamilian M, Anton P, de Riese W, Allhoff EP, Jonas U: Single potential analysis of cavernous electrical activity in impotent patients: a possible diagnostic method for autonomic cavernous dysfunction and cavernous smooth muscle degeneration. J Urol I99I, 146:77I-776.

17. Machtens SA, Stief CG, Gorek M, Becker AJ, Truss MC, Jonas U: Corpus cavernosum electromyography: technique and clinical implications. Tech Urol 1997, 3:147-I5I.

18. Kellner B, Stief CG, Hinrichs H, Hartung C: Computerized classification of corpus cavernosum electromyogram signals by the use of discriminant analysis and artificial neural networks to support diagnosis of erectile dysfunction. Urol Res 2000 , 28:6-13.

19. Shafik A, Shafik IA, El-Sibai O, Shafik AA: Overactive corpus cavernosum: a novel cause of erectile dysfunction. Andrologia 2004, 36:378-83.

20. Shafik A, Shafik AA, El-Sibai O, Shafik IA: Electrocavernosogram in erectile dysfunction: A diagnostic tool. Arch Androl 2004, 50:317-325.

21. Schellan TM: Induction of ejaculation by electrovibration. Ferti Steril 1968, 19:566-569.

22. Yokoyama O, Komatsu K, Kodama K, Yotsuyanagi S, Niikura S, Namiki M: Diagnostic value of intravesical lidocaine for overactive bladder. J Urol 2000, 164:340-343.

23. Silva C, Ribeiro MI, Cruz F: The effect of intravesical resiniferatoxin in patients with idiopathic detrusor instability suggests that involuntary detrusor contractions are triggered by $C$. fiber input. J Urol 2002, I68:575-580.

\section{Pre-publication history}

The pre-publication history for this paper can be accessed here:

http://www.biomedcentral.com/1471-2490/8/4/prepub

\begin{tabular}{|l|}
\hline Publish with Bio Med Central and every \\
scientist can read your work free of charge \\
"BioMed Central will be the most significant development for \\
disseminating the results of biomedical research in our lifetime. " \\
Sir Paul Nurse, Cancer Research UK \\
Your research papers will be: \\
• available free of charge to the entire biomedical community \\
• peer reviewed and published immediately upon acceptance \\
• cited in PubMed and archived on PubMed Central \\
• yours - you keep the copyright \\
Submit your manuscript here: \\
http://www.biomedcentral.com/info/publishing_adv.asp
\end{tabular}

